\title{
Embracing our Profession
}

$\mathrm{O}$ ver 35 years as a Registered Dietitian is a career milestone I proudly embrace, one many Dietitians of Canada (DC) members acknowledge surpassing! Beyond the reflection of those years, I look to a future where the general public widely accept the fact that, "Dietitians are the most credible, trustworthy source of nutrition advice"-DC's highest priority Ends Policy-one of four goals set for the organization with the aim to deliver the greatest impact.

With a visionary perspective and in collaboration, DC members have the competence to influence the direction of our profession's future. The Ends Policies preamble emphasizes a future where, "Policy makers and the public acknowledge that nutrition is a primary contributor to improving health and that the dietitian profession's unique body of knowledge and skills makes an integral contribution to health improvement at a cost that demonstrates the wise stewardship of resources."

As a result of the continuing evolution of technology we experience a world within a moment's reach, offering networking and research opportunities at the flick of a key and linkages with DC members, health professionals, and the public via teleconference and telehealth globally. In essence, "Dietitians, nutritionists and other health professionals have increased access to carefully selected and organized elements of the dietetic profession's unique knowledge base" - the third of four Ends policies.

It is my hope that you, in liaison with colleagues, embrace our profession, be a part of its evolving delivery of health services, and experience your own milestone.

To review the Ends Policies in its entirety contact board@ dietitians.ca

(Can J Diet Pract Res. 2017;78:1)

(DOI: 10.3148/cjdpr-2016-038)

Published at dcjournal.ca on 22 February 2017

\section{Valoriser notre profession}

$\mathrm{P}$ lus de 35 ans de carrière à titre de diététiste, voilà un important jalon que je partage fièrement avec plusieurs membres des Diététistes du Canada (DC)! Alors que je dresse le bilan de toutes ces années, j'envisage un avenir où le public reconnaîtra d'emblée que "les diététistes sont la source de conseils sur la nutrition la plus crédible et digne de confiance "- la priorité prédominante de la politique des DC - l'un des quatre objectifs définis par l'organisation pour maximiser les retombées.

En adoptant une perspective visionnaire et en misant sur la collaboration, les membres des DC ont les compétences nécessaires pour influer sur l'avenir de notre profession. Le préambule de la politique préconise un avenir où "les décideurs et le public reconnaissent que la nutrition est l'un des principaux facteurs contribuant à l'amélioration de la santé et que l'ensemble unique de connaissances et de compétences des diététistes contribue de manière importante à l'amélioration de la santé, et ce, à un coût qui démontre une gestion judicieuse des ressources."

En raison de l'évolution constante de la technologie, nous vivons dans un monde d'immédiateté où les possibilités de réseautage et de recherche sont à portée de clic et où les membres des DC, les professionnels de la santé et le public peuvent interagir au moyen de téléconférences et de la télésanté à l'échelle mondiale. En somme, "les diététistes, les nutritionnistes et autres professionnels de la santé ont un meilleur accès aux éléments soigneusement sélectionnés et organisés de l'ensemble unique de connaissances de la profession " - le troisième des quatre objectifs inscrits à la politique.

J'espère qu'en collaboration avec vos collègues, vous ferez honneur à la profession en participant activement à l'évolution de la prestation des services de santé et que votre carrière sera jalonnée de succès.

Pour consulter la politique dans son intégralité, écrivez à board@ dietitians.ca.

(Rev can prat rech diétét. 2017; 78:1)

(DOI: 10.3148/cjdpr-2016-038)

Publié au dcjournal.ca le 22 février 2017

\begin{tabular}{ll}
$\begin{array}{ll}\text { 2016-2017 } \\
\text { Board of } \\
\text { Directors }\end{array}$ & $\begin{array}{l}\text { Phyllis Marsch (Chair) } \\
\text { Winnipeg, MB } \\
\text { 204-275-1918 }\end{array}$ \\
\cline { 1 - 2 } $\begin{array}{l}\text { Conseil } \\
\text { d'administration }\end{array}$ & $\begin{array}{l}\text { Doug Cook } \\
\text { Toronto, ON } \\
416-413-9095\end{array}$
\end{tabular}

Colinda Hunter Edmonton, $\mathrm{AB}$ 780-577-3333

Sue Conlan Halifax, NS 902-697-2157
Nicole Spencer

Vancouver, BC 604-564-6534

Joane Routhier Kirkland, QC 514-398-7749

\author{
Judy Sheeshka \\ Surrey, BC \\ 778-294-3723 \\ Melissa Baker \\ Toronto, ON \\ 647-881-4576
}

Jean Helps Headingly, MB 204-837-3166

Chief Executive Officer Marsha Sharp 416-642-9314 